\title{
An approach version of fuzzy metric spaces including an ad hoc fixed point theorem
}

\author{
Antonio-Francisco Roldán-López-de-Hierro ${ }^{1 *}$, Manuel de la Sen², Juan Martínez-Moreno ${ }^{1}$ and \\ Concepción Roldán-López-de-Hierro ${ }^{3}$
}

\author{
"Correspondence: \\ afroldan@ujaen.es; aroldan@ugr.es \\ ${ }^{1}$ University of Jaén, Campus las \\ Lagunillas s/n, Jaén, 23071, Spain \\ Full list of author information is \\ available at the end of the article
}

\begin{abstract}
In this paper, inspired by two very different, successful metric theories such us the real view-point of Lowen's approach spaces and the probabilistic field of Kramosil and Michálek's fuzzy metric spaces, we present a family of spaces, called fuzzy approach spaces, that are appropriate to handle, at the same time, both measure conceptions. To do that, we study the underlying metric interrelationships between the above mentioned theories, obtaining six postulates that allow us to consider such kind of spaces in a unique category. As a result, the natural way in which metric spaces can be embedded in both classes leads to a commutative categorical scheme. Each postulate is interpreted in the context of the study of the evolution of fuzzy systems. First properties of fuzzy approach spaces are introduced, including a topology. Finally, we describe a fixed point theorem in the setting of fuzzy approach spaces that can be particularized to the previous existing measure spaces.
\end{abstract}

\section{Introduction}

The notion of metric has always been intimately related to a spatial conception. However, it was not until the end of the first decade of the twentieth century that Fréchet presented the currently accepted axioms on which this theory is based. After the appearance of the concept of metric space, and mainly due to its potential applications, many researchers have been interested in extending it to wider fields of knowledge. In this sense, in order to model real-world experiments, many theories have been introduced over the last century. In this manuscript we pay especial attention to two of them.

Some of the most productive extensions of the concept of metric were introduced in the field of probability. Convinced of the difficulty of accurately measuring the distance between two points in real life (for instance, between two subatomic particles), Menger proposed in 1942 the idea of a statistical metric space (see [1]). His main contribution was to replace the distance between two points, which had traditionally been a real number, by a random variable. Taking into account that, under uncertain environment, classical approaches are unable to give a complete answer to many real problems in several areas, Zadeh presented the notion of fuzzy set [2]. A number between 0 and 1 was employed to express the imprecision/vagueness that arises in many real situations. Subsequently, many authors, inspired by the notion of probabilistic metric space given by Schweizer and Sklar [3], have introduced their particular points of view about the definition of fuzzy metric space (see [4-8], among others), to which we will refer as FM-space. See also [9-11]. 
In 1975, Kramosil and Michálek [6] modified the axioms used until then and established a new class of FM-spaces provided with a Hausdorff topology. Later, other authors have considered certain modifications of this concept which are sufficient to verify new peculiar qualities (see [7]). FM-spaces have many applications. For example, in [12], the authors used FM-spaces in order to predict access histories working on variations of the fuzzy construction. In [13], Colubi and González-Rodríguez analyzed a fuzzifying process of finitely valued random variables by means of triangular fuzzy sets. Several studies of fuzzy regression analysis can be found in $[14,15]$. The concept of fuzzy number supposes a generalization of the notion of real number and there exists a complete arithmetic among them (see $[5,16])$. Furthermore, there exists a complete theoretic and practical analysis of fuzzy linear systems. In this field, inspired by Banach's theorem, fixed point theory has been intensively studied [17-31], even in frameworks like intuitionistic fuzzy metric spaces [32-36].

With respect to non-probabilistic extensions of the notion of metric space, the perspective can be highlighted introduced by Lowen through his approach spaces (in the sequel, A-spaces). Although they can seem, at first sight, simple generalizations of the necessary conditions that distances between point and subsets must satisfy, these spaces have turned out to have such good properties that they solved the problem of how to treat, in a unified way, such different notions as metrics, topologies, and uniformities, especially from the categorical view-point (see [37, 38]). In this sense, the main purpose for their introduction was to fill some gaps concerning categorical aspects of metrizable spaces. They are useful in many areas such as probability theory, functional analysis and function spaces. In his referential book [38], Lowen offered at least seven equivalent formulations of A-spaces.

With the aim of finding interrelationships between both successful theories, this paper introduces the notion of fuzzy approach space, which is a mixture of the best advantages of both conceptions. We also show some basic properties of this kind of spaces, and how the categories of quasi-metric spaces, A-spaces, and FM-spaces (in the sense of Kramosil and Michálek) can be naturally embedded in the supercategory of fuzzy approach spaces. Moreover, under some conditions, we provide these spaces with a topology. Finally, the study of contractions between fuzzy approach spaces leads to a first result in the field of fixed point theory involving this new class of spaces, which illustrates that some metric questions naturally arise in this ambiance.

\section{Preliminaries}

Let $\mathbb{N}=\{0,1,2, \ldots\}, \mathbb{R}$ and $\overline{\mathbb{R}}=[-\infty, \infty]$ denote the set of all non-negative integers, the set of all real numbers, and the extended real line, respectively (for simplicity, $+\infty$ will be denoted as $\infty)$. Given a real number $r \in[0, \infty$ [, we use the notation $t \geq r$ if $t \in[r, \infty], t>r$ if $t \in] r, \infty]$, and $t<\infty$ if $t \in[0, \infty[$.

In the sequel, $X$ will be a non-empty set and $\mathcal{P}(X)$ will denote the sets of all subsets of $X$. A fuzzy set $F$ on $X$ is a map $F: X \rightarrow[0,1]$. A fuzzy set $F$ is crisp if its image is included in $\{0,1\}$.

An (extended) metric space (briefly, $M$-space) is a set $X$ together with a function $\mathrm{d}: X \times$ $X \rightarrow \overline{\mathbb{R}}$ (called extended metric or distance function) satisfying the following properties:

M1. $\mathrm{d}(p, p)=0$ for all $p \in X$.

M2. $\mathrm{d}(p, q) \neq 0$ if $p \neq q$ for all $p, q \in X$.

M3. $\mathrm{d}(p, q)=\mathrm{d}(q, p)$ for all $p, q \in X$.

M4. Triangular inequality: $\mathrm{d}(p, q) \leq \mathrm{d}(p, x)+\mathrm{d}(x, q)$ for all $p, q, x \in X$. 
If M3 is omitted, the function d is a quasi-metric (briefly, qM-space). The topology of a qM-space $(X, \mathrm{~d})$ is completely determined by the distance function between two points. In a metric space, the distance between a point $p \in X$ and a subset $A$ of $X$ is the infimum of the distances between $p$ and all the points of the subset $A$. The analysis of the essential properties of a function $\delta: X \times \mathcal{P}(X) \rightarrow \overline{\mathbb{R}}$ such as the previous one (considering the empty infimum as $\infty$ ) leads to the following spaces.

Definition 2.1 (Lowen [37]) An approach space (briefly, $A$-space) is a pair $(X, \delta)$ where $X$ is a non-empty set and $\delta: X \times \mathcal{P}(X) \rightarrow \overline{\mathbb{R}}$ is a function satisfying the following properties:

A1. $\delta(p,\{p\})=0$ for all $p \in X$.

A2. $\delta(p, \varnothing)=\infty$ for all $p \in X$.

A3. $\delta(p, A \cup B)=\min (\delta(p, A), \delta(p, B))$ for all $p \in X$ and $A, B \in \mathcal{P}(X)$.

A4. $\delta(p, A) \leq \delta\left(p, A^{(s)}\right)+s$ for all $p \in X, A \in \mathcal{P}(X)$ and $s \in[0, \infty]$, where $A^{(s)}=\{q \in$ $X / \delta(q, A) \leq s\}$.

Lowen did a complete study of A-spaces in [38]. Condition A4 is analogous to the triangular inequality for these spaces. Under property A3, Lowen et al. [39] proved that condition A4 could be rewritten equivalently as follows:

A4' $\left(A^{(\alpha)}\right)^{(\beta)} \subseteq A^{(\alpha+\beta)}$ for all $A \in \mathcal{P}(X)$ and all $\alpha, \beta \in[0, \infty]$.

Lemma 2.1 (qM-space $\hookrightarrow$ A-space) Every $q M$-space $(X, \mathrm{~d})$ is an A-space $\left(X, \delta_{\mathrm{d}}\right)$ considering the function $\delta_{\mathrm{d}}: X \times \mathcal{P}(X) \rightarrow \overline{\mathbb{R}}$ given by:

$$
\forall x \in X, \forall A \in \mathcal{P}(X), \quad \delta_{\mathrm{d}}(x, A)= \begin{cases}\inf _{a \in A} \mathrm{~d}(x, a), & \text { if } A \neq \varnothing \\ \infty, & \text { if } A=\varnothing .\end{cases}
$$

A binary operation $*:[0,1] \times[0,1] \rightarrow[0,1]$ is a continuous $t$-norm if $([0,1], *)$ is a topological monoid with unity 1 such that $a * b \leq c * d$ whenever $a \leq c$ and $b \leq d$ for $a, b, c, d \in[0,1]$.

Definition 2.2 A fuzzy quasi-metric space in the sense of Kramosil and Michálek [6] (briefly, $\mathrm{F}_{q} \mathrm{M}$-space) is a triple $(X, M, *)$ where $X$ is a non-empty set, $*$ is a continuous $t$-norm and $M: X \times X \times[0, \infty[\rightarrow[0,1]$ is a fuzzy map such that, for all $x, y, z \in X$ :

FM1. $M(x, y, 0)=0$.

FM2. $x=y$ if, and only if, $M(x, y, t)=1$ for all $0<t<\infty$.

FM3. $M(x, z, t+s) \geq M(x, y, t) * M(y, z, s)$ for all $0 \leq s, t<\infty$.

FM4. $M(x, y, \cdot):[0, \infty[\rightarrow[0,1]$ is left-continuous.

If we additionally assume that $M$ satisfies the condition of symmetry:

$$
M(x, y, t)=M(y, x, t), \quad \text { for all } x, y \in X \text { and } 0<t<\infty
$$

then $(X, M, *)$ is called a fuzzy metric space (FM-space).

We point out that Kramosil and Michálek introduced a slightly different definition. If $M(x, y, t)$ is interpreted as the probability of the distance between $x$ and $y$ to be less than or 
equal to $t$, and inspired by the probabilistic framework of random variables, they assumed that

$$
\lim _{t \rightarrow \infty} M(x, y, t)=1 \quad \text { for all } x, y \in X .
$$

However, the previous one is the most extended way to handle their fuzzy spaces.

Remark 2.1 In order to define a Hausdorff topology and to prove some existing results (including Baire's theorem), George and Veeramani [7] slightly modified the concept of fuzzy metric space introduced by Kramosil and Michálek. It is well known that all fuzzy (quasi-)metric spaces in the sense of George and Veeramani are also fuzzy (quasi-)metric spaces in the sense of Kramosil and Michálek. Hence, the results we will prove also include fuzzy metric spaces in the sense of George and Veeramani (see also [40]).

Proposition 2.1 (Grabiec [41]) For all $x, y \in X, M(x, y, \cdot)$ is a nondecreasing function on $[0, \infty[$.

Proof If $z=y$ and $s>0$ in FM3, we have $M(x, y, t+s) \geq M(x, y, t) * M(y, y, s)=M(x, y, t) * 1=$ $M(x, y, t)$.

If $(X, \mathrm{~d})$ is a $M$-space, we can consider the fuzzy set $M_{s}: X \times X \times[0, \infty[\rightarrow[0,1]$ given by $M_{s}(x, y, 0)=0$ and

$$
\left.M_{s}(x, y, t)=\frac{t}{t+\mathrm{d}(x, y)}, \quad \text { if } t \in\right] 0, \infty[
$$

for all $x, y \in X$ and all $t \in\left[0, \infty\left[\right.\right.$. If $*=\cdot$ is the usual multiplication in [0,1], then $\left(X, M_{s}, \cdot\right)$ is a FM-space, called standard fuzzy metric space [7]. The following lemma presents an alternative way to view a M-space as a FM-space.

Lemma 2.2 (qM-space $\hookrightarrow \mathrm{F}_{q} \mathrm{M}$-space) Every $q M$-space $(X, \mathrm{~d})$ is a $F_{q} M$-space $\left(X, M_{\mathrm{d}}\right.$, min) considering the continuous $t$-norm $*=\min$ and the fuzzy set $M_{\mathrm{d}}: X \times X \times[0, \infty[\rightarrow[0,1]$ given, for any $x, y \in X$ and $t \in[0, \infty[$, by

$$
\begin{aligned}
& \text { if } \mathrm{d}(x, y)<\infty, \quad M_{\mathrm{d}}(x, y, t)= \begin{cases}0, & \text { if } 0 \leq t \leq \mathrm{d}(x, y), \\
1, & \text { if } \mathrm{d}(x, y)<t<\infty ;\end{cases} \\
& \text { if } \mathrm{d}(x, y)=\infty, \quad M_{\mathrm{d}}(x, y, t)=0 \quad \text { for all } t<\infty .
\end{aligned}
$$

Proof FM1, FM2, and FM4 are trivial. Let $x, y, z \in X$ be three different points. Note that

$$
\begin{aligned}
M_{\mathrm{d}}(x, y, t) * M_{\mathrm{d}}(y, z, s)= & \min \left(M_{\mathrm{d}}(x, y, t), M_{\mathrm{d}}(y, z, s)\right) \\
= & \min \left(\left\{\begin{array}{ll}
0, & \text { if } t \leq \mathrm{d}(x, y)<\infty \text { or } t<\mathrm{d}(x, y)=\infty, \\
1, & \text { if } \mathrm{d}(x, y)<t<\infty,
\end{array}\right\},\right. \\
& \left.\left\{\begin{array}{ll}
0, & \text { if } s \leq \mathrm{d}(y, z)<\infty \text { or } s<\mathrm{d}(y, z)=\infty, \\
1, & \text { if } \mathrm{d}(y, z)<s<\infty,
\end{array}\right\}\right) \\
= & \begin{cases}1, & \text { if } \mathrm{d}(x, y)<t<\infty \text { and } \mathrm{d}(y, z)<s<\infty, \\
0, & \text { in any other case. }\end{cases}
\end{aligned}
$$


To prove FM3, we can reduce to consider the cases $\mathrm{d}(x, y)<t<\infty$ and $\mathrm{d}(y, z)<s<$ $\infty$. Then $t+s>\mathrm{d}(x, y)+\mathrm{d}(y, z) \geq \mathrm{d}(x, z)$, so $M_{\mathrm{d}}(x, z, t+s)=1$. This proves that if $\min \left(M_{\mathrm{d}}(x, y, t), M_{\mathrm{d}}(y, z, s)\right)=1$, then $M_{\mathrm{d}}(x, z, t+s)=1$, and FM3 is true.

\section{Fuzzy approach spaces}

In this section, the notion of approach space is generalized to the fuzzy setting and fuzzy approach spaces are presented.

Definition 3.1 A fuzzy approach space (briefly, FA-space) is a pair $(X, F)$ where $X$ is a set and $F: X \times \mathcal{P}(X) \times[0, \infty] \rightarrow[0,1]$ is a fuzzy set satisfying the following conditions, for all $x \in X$, all $A, B \in \mathcal{P}(X)$ and all $s, t \in[0, \infty]$ :

FA1. $F(x, A, 0)=0$.

FA2. $F(x, \varnothing, t)=0$ for all $t<\infty$.

FA3. $F(x,\{x\}, t)=1$ for all $t>0$.

FA4. $F(x, A, \cdot):[0, \infty[\rightarrow[0,1]$ is a left-continuous function.

FA5. $F(x, A \cup B, t) \geq \max (F(x, A, t), F(x, B, t))$.

FA6. If we denote, for all $r \in[0, \infty[$,

$$
\begin{aligned}
& A^{(r)}=\{y \in X / F(y, A, t)=1, \forall t>r\} \quad \text { and } \\
& A^{(\infty)}=\{y \in X / F(y, A, \infty)=1\},
\end{aligned}
$$

then

$$
\begin{aligned}
& F(x, A, t+s) \geq F\left(x, A^{(r)}, t\right) \quad \text { for all } r \in[0, s[\text { and } \\
& F(x, A, \infty) \geq F\left(x, A^{(\infty)}, t\right) .
\end{aligned}
$$

If FA6 is omitted, then $(X, F)$ is a fuzzy semi-approach space (briefly, $F_{s} A$-space). The map $F$ is symmetric (or the $\mathrm{F}_{s} \mathrm{~A}$-space $(X, F)$ is symmetric) if $F(x,\{y\}, t)=F(y,\{x\}, t)$ for all $x, y \in X$ and all $t>0$. Finally, $(X, F)$ is a crisp FA-space (or a crisp $F_{s} \mathrm{~A}$-space) if the image of $F$ is in $\{0,1\}$.

Remark 3.1 In broad terms, considering $s=\infty$ in the first inequality of FA6 property and joining with the second inequality, we have

$$
F(x, A, \infty) \geq F\left(x, A^{(r)}, t\right), \quad \text { for all } r, t \in[0, \infty] .
$$

Remark 3.2 The original definition of approach space establishes equality in condition A3. However, it will be replaced in FA5 with inequality. In fact, it would be possible to develop a similar theory for approach spaces by setting in A3 only the inequality $\geq$.

Kramosil and Michálek considered $M(x, y, t)$ defined only for $t \in[0, \infty[$. In our definition of a fuzzy approach space, $\infty$ must be included trying to generalize the approach spaces. But it is not a problem because we can consider, in Definition 2.2, $M(x, y, \infty)=1$ for all $x, y \in X$.

When $A=\{y\}$ is a single point, we denote $F(x,\{y\}, t)$ by $F(x, y, t)$, and, similarly, we will use this notation with other maps. 
The following examples are given to illustrate a possible interpretation of the previous conditions in order to study the dynamic of a fuzzy system that can appear in fuzzy decision making.

Example 3.1 Let $X$ be a set of experts that express their opinions about one or more questions. For instance, one may consider the level of satisfaction with respect to a product that is going to come on to the market. This level can be expressed as a number between 0 and 1. Experts discuss among themselves and, in the course of time, they reach agreement about their points of view. In this way, an expert's opinion is not constant in time and it changes according the other experts' opinions. Then it seems reasonable to measure the 'degree of agreement (or disagreement) between an expert $x \in X$ and a experts' committee $A \subseteq X$ in the instant $t$ '. This uncertainty can be interpreted as the difference between 1 and the minor distance between the expert's opinion $x$ (a number between 0 and 1 ) and the set of opinions of the rest of members of $A$.

This interpretation leads to a fuzzy set on $X \times P(X) \times[0, \infty]$, which could be a fuzzy approach space when the following properties are satisfied:

- Property FA1 means that, at the beginning, there is not agreement between the experts.

- Property FA2 establishes that it is necessary, at least, for a person in the committee to reach an agreement.

- Property FA3 says us that an expert's opinion is always coherent with himself/herself.

- Property FA4 shows that an expert's opinion depends continuously on his/her opinion in the past, but not in the future.

- Property FA5 guarantees that there being involved more participants, it will be easier to persuade an expert to reach an agreement with the rest.

Previous properties are certainly natural. However, condition FA6 shows us that the system evolves in the following way. If $A \subseteq X$ is an expert committee and $r \in[0, \infty[$ is an instant, the closure

$$
\left.\left.A^{(r)}=\{y \in X / F(y, A, t)=1, \forall t \in] r, \infty\right]\right\}
$$

can be interpreted as the set of experts that are capable of reaching an agreement with the members of the committee $A$ before an instant $r$, that is, the set of experts that the committee $A$ could persuade before $r$ units of time. In this case, condition FA6,

$$
F(x, A, t+s) \geq F\left(x, A^{(r)}, t\right)
$$

means that, in the instant $t+s$ (that is, spending $s$ units of time), an expert $x$ and the committee $A$ have reached a bigger agreement than the agreement reached by the expert $x$ with the committee $A^{(r)}$ in the instant $t$.

The next example is inspired by the previous one.

Example 3.2 Let $X$ a set of three experts, that is, $X=\{x, y, z\}$. Let $F: X \times P(X) \times[0, \infty] \rightarrow$ $[0,1]$ be the map defined as follows (only for non-trivial cases):

$$
F(x, y, t)=F(x,\{y, z\}, t)=F(y,\{x, z\}, t)= \begin{cases}0, & \text { if } t=0 \\ 1, & \text { if } t>0\end{cases}
$$




$$
F(x, z, t)=F(y, z, t)=F(z,\{x, y\}, t)= \begin{cases}0, & \text { if } t=0 \\ 1 / 2, & \text { if } 0<t \leq 1 \\ 1, & \text { if } t>1\end{cases}
$$

It is easy to show that $(X, F)$ is a symmetric FA-space. The fuzzy system evolves in the following way: experts $x$ and $y$ always agree among themselves, but there is a certain discrepancy with the expert $z$. During a period of time, they discuss and their postures come closer to coincide in a $50 \%$. When a unity of time is spent, the agreement between the three experts is complete.

\section{Two families of FA-spaces and a commutative diagram}

Firstly, we will show that there are two families of spaces satisfying the above six properties.

Let $d: X \times X \rightarrow[0, \infty]$ be a map, $x \in X, A \in \mathcal{P}(X)$ and $t \in[0, \infty]$. Although $d$ is not necessarily a metric or a quasi-metric, it is easy to see that

$$
\sup _{a \in A}\left\{\begin{array}{ll}
0, & \text { if } t \leq d(x, a), \\
1, & \text { if } t>d(x, a)
\end{array}\right\}= \begin{cases}0, & \text { if } t \leq \inf _{a \in A} d(x, a), \\
1, & \text { if } t>\inf _{a \in A} d(x, a)\end{cases}
$$

even if $\inf _{a \in A} d(x, a)=\infty$ (replacing $\leq$ by $\left.<\right)$ or $A=\varnothing$ (considering $\left.\inf _{a \in \varnothing} d(x, a)=\infty\right)$. See Proposition A.1 in the Appendix.

Taking into account the transformation from M-spaces to FM-spaces, associated with an approach space $(X, \delta)$, the following FA-space can be built:

$$
F^{\delta}(x, A, t)= \begin{cases}0, & \text { if } t=0 \\ \frac{t}{t+\delta(x, A)}, & \text { if } 0<t<\infty \\ 1, & \text { if } t=\infty\end{cases}
$$

However, for our purposes, the following structure would be more interesting.

Theorem 4.1 (A-space $\hookrightarrow$ FA-space) Let $(X, \delta)$ be an A-space. We define a fuzzy set $F_{\delta}$ on $X \times \mathcal{P}(X) \times[0, \infty]$ given in the following way:

$$
\begin{aligned}
& \text { if } \delta(x, A)<\infty, \quad F_{\delta}(x, A, t)= \begin{cases}0, & \text { if } t \leq \delta(x, A), \\
1, & \text { if } t>\delta(x, A) ;\end{cases} \\
& \text { if } \delta(x, A)=\infty, \quad F_{\delta}(x, A, t)= \begin{cases}0, & \text { if } t<\infty, \\
1, & \text { if } t=\infty .\end{cases}
\end{aligned}
$$

Then $\left(X, F_{\delta}\right)$ is a crisp FA-space, satisfying the equality in $F A 5$, and $A^{(\infty)}=X$ for all $A \in$ $\mathcal{P}(X)$.

Proof FA1, FA2, FA3, and FA4 are immediate and clearly $\operatorname{Im} F_{\delta}=\{0,1\}$. In order to prove FA5, if $A, B \subseteq X$, we can suppose $\delta(x, A \cup B)=\min (\delta(x, A), \delta(x, B))=\delta(x, A) \leq \delta(x, B)$. If $\delta(x, B)<\infty$, then

$$
\begin{aligned}
& \max \left(F_{\delta}(x, A, t), F_{\delta}(x, B, t)\right) \\
& \quad=\max \left(\left\{\begin{array}{ll}
0, & \text { if } t \leq \delta(x, A)<\infty, \\
1, & \text { if } \delta(x, A)<t<\infty,
\end{array}\left\{\begin{array}{ll}
0, & \text { if } t \leq \delta(x, B)<\infty, \\
1, & \text { if } \delta(x, B)<t<\infty
\end{array}\right)\right.\right.
\end{aligned}
$$




$$
\begin{aligned}
& =\left\{\begin{array}{ll}
1, & \text { if } \delta(x, A)<t<\infty \text { or } \delta(x, B)<t<\infty, \\
0, & \text { in any other case }
\end{array}\right\} \\
& =\left\{\begin{array}{ll}
1, & \text { if } t>\min (\delta(x, A), \delta(x, B)), \\
0, & \text { in any other case }
\end{array}\right\} \\
& =\left\{\begin{array}{ll}
1, & \text { if } t>\delta(x, A \cup B), \\
0, & \text { in any other case }
\end{array}\right\}=F_{\delta}(x, A \cup B, t) .
\end{aligned}
$$

Other cases are similar. See Remark A.2 in the Appendix for more details. In any case, $\left(X, F_{\delta}\right)$ satisfies the equality in FA5.

For every $r \in[0, \infty]$, the set $A^{(r)}$ is the same as an A-space as a FA-space, since, for $r<\infty$,

$$
\begin{aligned}
A_{\mathrm{FA}-\text { space }}^{(r)} & =\left\{x \in X / F_{\delta}(x, A, t)=1, \forall t>r\right\}=\{x \in X / t>\delta(x, A), \forall t>r\} \\
& =\{x \in X / \delta(x, A) \leq r\}=A_{\mathrm{A} \text {-space }}^{(r)}
\end{aligned}
$$

If $r=\infty$, as $F_{\delta}(x, A, \infty)=1$ for all $x \in X$ and all $A \in \mathcal{P}(X)$, we have $A^{(\infty)}=X$ for all $A \in \mathcal{P}(X)$. Then $A_{\mathrm{A} \text {-space }}^{(\infty)}=X=A_{\mathrm{FA} \text {-space }}^{(\infty)}$. For FA6, we are going to prove that if $F_{\delta}\left(x, A^{(r)}, t\right)=1$, then $F_{\delta}(x, A, t+s)=1$. In the finite case $t, s \in\left[0, \infty\left[\right.\right.$, if $F\left(x, A^{(r)}, t\right)=1$, then $t>\delta\left(x, A^{(r)}\right)$. If $s>r$, then $t+s>t+r>\delta\left(x, A^{(r)}\right)+r \geq \delta(x, A)$. Therefore, $F_{\delta}(x, A, t+s)=1$. See Remark A.3 in the Appendix. This completes the proof.

Theorem 4.2 ( $\mathrm{F}_{q} \mathrm{M}$-space $\hookrightarrow$ FA-space $)$ Let $X$ be a set and let $M: X \times X \times[0, \infty[\rightarrow[0,1]$ be any fuzzy set satisfying FM1, FM4, and $M(x, x, t)=1$ for all $x \in X$ and all $t>0$. Let $F_{M}: X \times \mathcal{P}(X) \times[0, \infty] \rightarrow[0,1]$ be the fuzzy set given by

$$
F_{M}(x, A, t)= \begin{cases}0, & \text { if } A=\varnothing \text { and } t<\infty \\ \sup _{a \in A} M(x, a, t), & \text { if } A \neq \varnothing \text { and } t<\infty \\ 1, & \text { if } t=\infty\end{cases}
$$

Then $\left(X, F_{M}\right)$ is a $F_{S} A$-space that verifies the equality in $F A 5$ and $A^{(\infty)}=X$ for all $A \in \mathcal{P}(X)$. Moreover, if $(X, M, *)$ is a $F_{q} M$-space, then $\left(X, F_{M}\right)$ is a FA-space, $\left.F_{M}\right|_{X \times X \times[0, \infty[}=M$ and satisfies the following property:

- If $x, y \in X$ verify that $F_{M}(x, y, t)=1$ for every $0<t<\infty$, then $x=y$.

Proof FA1 and FA2 are a direct consequence of the definition of $F$, FA3 is obtained from the hypothesis $M(x, x, t)=1$ for all $x \in X$ and all $t>0$, and FA4 is obtained from FM4, since $F_{M}(x, y, t)=M(x, y, t)$ for all $0<t<\infty$ and the supremum of left-continuous functions in $[0, \infty$ [ is a left-continuous function too. To prove the equality in FA5, let $A, B \in \mathcal{P}(X)$. If $A=\varnothing$, then

$$
\begin{aligned}
\max \left(F_{M}(x, A, t), F_{M}(x, B, t)\right) & =\max \left(\left\{\begin{array}{ll}
0, & \text { if } t<\infty, \\
1, & \text { if } t=\infty,
\end{array} F_{M}(x, B, t)\right)\right. \\
& =F_{M}(x, B, t)=F_{M}(x, A \cup B, t) .
\end{aligned}
$$


Suppose that $A, B \neq \varnothing$. If $t=0$ or $t=\infty$, we trivially have the equality. If $0<t<\infty$, the equality

$$
\sup _{z \in A \cup B} M(x, z, t)=\max \left(\sup _{a \in A} M(x, a, t), \sup _{b \in B} M(x, b, t)\right)
$$

implies that $F_{M}(x, A \cup B, t)=\max \left(F_{M}(x, A, t), F_{M}(x, B, t)\right)$. In any case, $(X, F)$ verifies the equality in FA5. So $(X, F)$ is a $\mathrm{F}_{s} \mathrm{~A}$-space.

Now, suppose that $(X, M, *)$ is an $\mathrm{F}_{q} \mathrm{M}$-space. Let us show that $(X, F)$ satisfies property FA6. Let $x \in X, A \in P(X)$ and $r \in[0, \infty]$. If $A=\varnothing$, the condition FA6 is trivial. It is sufficient to show that the property FA6 holds true for every $A \neq \varnothing$. Since, for all $x, y, z \in X$ and all $0<t, s<\infty$,

$$
M(x, z, t+s) \geq M(x, y, t) * M(y, z, s)
$$

in particular,

$$
M(x, y, t+s) \geq M(x, a, t) * M(a, y, s)
$$

for all $x \in X, a \in A^{(r)}, y \in A, t>0$, and $s>r \geq 0$. Taking the supremum over $y \in A$, then

$$
\begin{aligned}
F_{M}(x, A, t+s) & =\sup _{y \in A} M(x, y, t+s) \geq M(x, a, t) * \sup _{y \in A} M(a, y, s) \\
& =M(x, a, t) * F_{M}(a, A, s) .
\end{aligned}
$$

Since $a \in A^{(r)}$, we have $F_{M}(a, A, s)=1$, for $s>r$. Thus, $F_{M}(x, A, t+s) \geq M(x, a, t)$. Taking the supremum over $a \in A^{(r)}$, we have $F_{M}(x, A, t+s) \geq F_{M}\left(x, A^{(r)}, t\right)$. So $(X, F)$ is a FA-space. Finally, the last property is a consequence of FM2.

The last property in the previous theorem will be really interesting in the sequel and we will refer to it as FA7.

FA7. If $x, y \in X$ are such that $F(x, y, t)=1$ for every $0<t<\infty$, then $x=y$.

Corollary 4.1 The following diagram commutes in the category of sets, that is, $F_{\delta_{d}}=F_{M_{d}}$ :

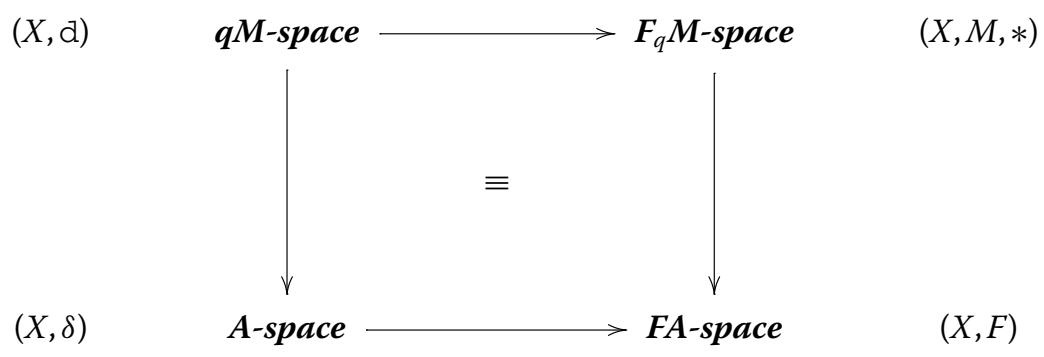

Therefore, if $(X, \mathrm{~d})$ is a $q M$-space, then $\left(X, F_{\delta_{d}}\right)$ is a crisp $F A$-space which verifies property $F A 7$, satisfies the equality in $F A 5$, and $A^{(\infty)}=X$ for all $A \in \mathcal{P}(X)$. 
Proof Let $(X, \mathrm{~d})$ be a qM-space. Then $X$ is an A-space if we define $\delta_{\mathrm{d}}$ as in Lemma 2.1. As a FA-space, the fuzzy map $F_{\delta_{\mathrm{d}}}$ is:

$$
\begin{aligned}
& \text { if } \delta_{\mathrm{d}}(x, A)=\inf _{a \in A} \mathrm{~d}(x, a)<\infty, \quad F_{\delta_{\mathrm{d}}}(x, A, t)= \begin{cases}0, & \text { if } t \leq \delta_{\mathrm{d}}(x, A), \\
1, & \text { if } t>\delta_{\mathrm{d}}(x, A) ;\end{cases} \\
& \text { if } \delta_{\mathrm{d}}(x, A)=\inf _{a \in A} \mathrm{~d}(x, a)=\infty, \quad F_{\delta_{\mathrm{d}}}(x, A, t)= \begin{cases}0, & \text { if } t<\infty, \\
1, & \text { if } t=\infty .\end{cases}
\end{aligned}
$$

On the other hand, $(X, \mathrm{~d})$ is a $\mathrm{F}_{q} \mathrm{M}$-space $\left(X, M_{\mathrm{d}}, \mathrm{min}\right)$ as in Lemma 2.2 , and then $F_{M_{\mathrm{d}}}$ is given by

$$
F_{M_{\mathrm{d}}}(x, A, t)= \begin{cases}0, & \text { if } t=0 \text { or }(A=\varnothing \text { and } t<\infty), \\ \sup _{a \in A} M_{\mathrm{d}}(x, a, t), & \text { if } A \neq \varnothing \text { and } 0<t<\infty, \\ 1, & \text { if } t=\infty\end{cases}
$$

It is easy to see that if $A=\varnothing$ or $\delta_{\mathrm{d}}(x, A)=\infty$ (in this case, $\mathrm{d}(x, a)=\infty$ for all $a \in A$ ), then

$$
F_{\delta_{\mathrm{d}}}(x, A, t)=\left\{\begin{array}{ll}
0, & \text { if } t<\infty, \\
1, & \text { if } t=\infty
\end{array}\right\}=F_{M_{\mathrm{d}}}(x, A, t) .
$$

It is always true that $F_{\delta_{\mathrm{d}}}(x, A, 0)=0=F_{M_{\mathrm{d}}}(x, A, 0)$ and $F_{\delta_{\mathrm{d}}}(x, A, \infty)=1=F_{M_{\mathrm{d}}}(x, A, \infty)$. Suppose that $A \neq \varnothing, \delta_{\mathrm{d}}(x, A)<\infty$ and $0<t<\infty$. Then, by property (4)

$$
\begin{aligned}
F_{M_{\mathrm{d}}}(x, A, t) & =\sup _{a \in A} M_{\mathrm{d}}(x, a, t)=\sup _{a \in A}\left\{\begin{array}{ll}
0, & \text { if } t \leq \mathrm{d}(x, a), \\
1, & \text { if } \mathrm{d}(x, a)<t
\end{array}\right\} \\
& =\left\{\begin{array}{ll}
0, & \text { if } t \leq \inf _{a \in A} \mathrm{~d}(x, a)<\infty, \\
1, & \text { if } \inf _{a \in A} \mathrm{~d}(x, a)<t<\infty
\end{array}\right\}=F_{\delta_{\mathrm{d}}}(x, A, t) .
\end{aligned}
$$

So $F_{\delta_{\mathrm{d}}}=F_{M_{\mathrm{d}}}$ in any case and the diagram commutes.

\section{Characterization of FA-spaces}

Now, we ask about the fuzzy maps $F: X \times \mathcal{P}(X) \times[0, \infty] \rightarrow[0,1]$ that could provide $X$ with an FA-space structure. In these cases, it could be interesting to weaken the conditions FA5 and FA6.

Proposition 5.1 Let $X$ be a set, and $F: X \times \mathcal{P}(X) \times[0, \infty] \rightarrow[0,1]$ be a fuzzy set satisfying the conditions $F A 1$ and $F A 2$ such that:

- $F(x, A, \infty)=1$ for all $x \in X$ and all $A \in \mathcal{P}(X)$.

- $F(x, A, t)=1$ for all $x \in A \subseteq X$ and all $t>0$.

Then property $F A 5$ is equivalent to

FA5'. For every non-empty subsets $A, B \subseteq X$ such that $A \neq B$ and $A \cup B \subset X$, for all $t>0$ and for all $x \in X \backslash(A \cup B)$, we have $F(x, A \cup B, t) \geq \max (F(x, A, t), F(x, B, t))$.

The equality in FA5 is always true if, and only if, the equality is achieved in the previous inequality.

Furthermore, condition FA6 is equivalent to 
FA6'. For all $t, s \in] 0, \infty[$, all $r \in[0, s[$, all $\varnothing \neq A \subset X$ and all $x \in X \backslash A$, the following inequality holds: $F(x, A, t+s) \geq F\left(x, A^{(r)}, t\right)$.

Proof We are going to see that there exist some cases in which, under the above mentioned hypotheses, the inequality (or the equality) trivially holds.

Property $F A 5^{\prime}$. If $A$ is empty, then $F(x, A \cup B, t)=F(x, B, t)$ and, by condition FA2, for all $t<\infty$

$$
\begin{aligned}
\max (F(x, A, t), F(x, B, t)) & =\max (F(x, \varnothing, t), F(x, B, t))=\max (0, F(x, B, t)) \\
& =F(x, B, t)=F(x, A \cup B, t),
\end{aligned}
$$

so we get the equality. If $t=\infty$, then the two last terms of the above identities are 1 . Suppose that $A$ and $B$ are non-empty. If $x \in A \cup B$, then, by hypothesis, as $x \in A$ or $x \in B$,

$$
F(x, A \cup B, t)=\left\{\begin{array}{ll}
0, & \text { if } t=0, \\
1, & \text { if } t>0
\end{array}\right\}=\max (F(x, A, t), F(x, B, t)) \text {, }
$$

and the equality holds. If $A \cup B=X$, then $x \in A \cup B$, and this case leads to the equality. Therefore, we can reduce the inequality to the case in which $\varnothing \neq A \cup B \subset X$ and $x \in X \backslash(A \cup B)$. If $A=B$, the equality is trivial. In all of these cases, the equality holds. So the equality in FA5 is equivalent to the equality in FA5'.

Property $F A 6^{\prime}$. If $t=0$, by FA1, $F\left(x, A^{(r)}, 0\right)=0$, and the inequality is obvious. The condition is empty if $s=0$, because $r \in\left[0, s\left[\right.\right.$. If $t=\infty$ or $s=\infty$, then $F(x, A, \infty)=1 \geq F\left(x, A^{(\infty)}, t\right)$. If $A=\varnothing$ then, by property FA2,

$$
\varnothing^{(r)}=\{x \in X / F(x, \varnothing, t)=1, \forall t>r\}=\varnothing, \quad \text { for all } r<\infty,
$$

since $r<s \leq \infty$, so $F\left(x, \varnothing^{(r)}, t\right)=F(x, \varnothing, t)=0$ for all $t \in[0, \infty[$. If $x \in A$, we suppose that $F(x, A, t)=1$ for all $t>0$, so $F(x, A, t+s)=1 \geq F\left(x, A^{(r)}, t\right)$ for all $t, s>0$ and $r \in[0, s[$. If $A=X$, then $x \in A$. So, we can reduce the inequality to the cases in which $\varnothing \neq A \subset X$, $x \in X \backslash A$, and $t, s \in] 0, \infty[$.

\section{First properties of the FA-spaces}

The following definition is possible even if the set $X$ has not been provided with a topology. In fact, in Section 7, we will show how to consider an appropriate topology on each FAspace depending on the fuzzy map $F$.

Definition 6.1 The closure $\bar{A}$ of a subset $A$ of a $\mathrm{F}_{s} \mathrm{~A}$-space $(X, F)$ is

$$
\bar{A}=A^{(0)}=\{x \in X / F(x, A, t)=1, \forall t>0\} .
$$

We say that $A$ is closed if $\bar{A} \subseteq A$.

The following properties hold even if $F$ does not satisfy the condition FA6.

Lemma 6.1 Let $(X, F)$ be a $F_{s} A$-space, $x, a \in X, t, r, s \in[0, \infty]$, and $A, B \in \mathcal{P}(X)$. 
(1) If $a \in A$, then $F(a, A, t)=1$ for all $t>0$.

(2) If $a \in A$, then $F(x, a, t) \leq F(x, A, t)$ for all $t \geq 0$.

(3) If $A \subseteq B \subseteq X$, then $F(x, A, t) \leq F(x, B, t)$ for all $t \geq 0$.

(4) If $A \neq \varnothing$, $\sup _{a \in A} F(x, a, t) \leq F(x, A, t)$ for all $t \geq 0$.

(5) If $A \subseteq B \subseteq X$, then $A^{(r)} \subseteq B^{(r)}$ for all $r \geq 0$.

(6) If $0 \leq r \leq s \leq \infty$, then

$$
A \subseteq \bar{A}=A^{(0)} \subseteq A^{(r)} \subseteq A^{(s)} \subseteq \cdots \subseteq A^{(\infty)} \subseteq X
$$

In particular, $F(x, A, t) \leq F(x, \bar{A}, t)$ for all $t \geq 0$.

(7) $A$ is closed if, and only if, $\bar{A}=A$.

(8) $\varnothing^{(s)}=\varnothing$ for all $s \in[0, \infty[$.

(9) $x \in A^{(r)}$ if, and only if, $x \in A^{(r+s)}$ for every $s>0$, i.e.,

$$
A^{(r)}=\bigcap_{s>r} A^{(s)}=\bigcap_{s>0} A^{(r+s)} .
$$

(10) If $\Delta(x, A)=\left\{s \in\left[0, \infty\left[/ x \in A^{(s)}\right\}\right.\right.$ is a non-empty set, then there exists $a \in[0, \infty[$ such that $\Delta(x, A)=[a, \infty$ [. If $\Delta(x, A) \neq \varnothing$ then $\inf \Delta(x, A)=\min \Delta(x, A)$.

(11) The following properties are equivalent:

(a) $A^{(\infty)}=X$ for all $A \in \mathcal{P}(X)$.

(b) $F(x, A, \infty)=1$ for all $x \in X$ and all $A \in \mathcal{P}(X)$.

(c) $F(x, \varnothing, \infty)=1$ for all $x \in X$.

(d) $\varnothing^{(\infty)}=X$.

Proof (1) By conditions FA3 and FA5, if $a \in A$, we have, for $t>0$,

$$
F(a, A, t)=F(a, A \cup\{a\}, t) \geq \max (F(a, A, t), F(a, a, t))=\max (F(a, A, t), 1)=1 .
$$

(2) It follows from condition FA5.

(3) If $A \subseteq B$, then $A \cup B=B$, and thus:

$$
F(x, B, t)=F(x, A \cup B, t) \geq \max (F(x, A, t), F(x, B, t)) \geq F(x, A, t), \quad \text { for all } t \in[0, \infty] .
$$

(4) By item (2), we find that if $a \in A$, then $F(x, a, t) \leq F(x, A, t)$. Taking the supremum, $\sup _{a \in A} F(x, a, t) \leq F(x, A, t)$.

(5) If $A \subseteq B$ and $r<\infty$, by item (3), $F(x, A, t) \leq F(x, B, t)$ for each $t \geq 0$. If $x \in A^{(r)}$, that is, $F(x, A, t)=1$ for each $t>r$, we have $F(x, B, t)=1$ for each $t>r$, i.e., $x \in B^{(r)}$. If $r=\infty$, a similar argument can be used.

(6) If $a \in A$, by item (1), $F(a, A, t)=1$ for each $t>0$. Thus $a \in \bar{A}$. Let $r \leq s$. If $x \in A^{(r)}$, i.e., $F(x, A, t)=1$, for each $t>r$, then, in particular, $F(x, A, t)=1$ for each $t>s$. Thus $x \in A^{(s)}$. A similar argument is valid if $r=\infty$ or $s=\infty$. See Remark A.4 in the Appendix.

(7) It is trivial since $A \subseteq \bar{A}$.

(8) From FA2, $F(x, \varnothing, t)=0$ for all $t<\infty$. So $\varnothing^{(s)}=\{x \in X / F(x, \varnothing, t)=1, \forall t>s\}=\varnothing$ if $s<\infty$.

(9) By definition, $x \in A^{(r)}$ if, and only if, $F(x, A, t)=1$ for all $t>r$. This condition is equivalent to the following: for each $s \in] r, \infty$ [, we see that $F(x, A, t)=1$ holds for all $t \in] r, \infty$, i.e., $x \in A^{(r+s)}$ for all $s>0$. 
(10) Let $r \in \Delta(x, A)$, that is, $x \in A^{(r)}$. From the previous item, $x \in \bigcap_{s>r} A^{(s)}$, i.e., $x \in A^{(s)}$ for all $s>r$. So $[r, \infty[\subseteq \Delta(x, A)$. This proves that $\Delta(x, A)$ is a real non-bounded interval. Let $a=\inf \Delta(x, A)$. It is clear that $0 \leq a<\infty$. For all $s>a$ we have $s \in \Delta(x, A)$, that is, $x \in A^{(s)}$. The previous item implies that $x \in A^{(a)}$, so $a \in \Delta(x, A)$ and we deduce that $\Delta(x, A)=[a, \infty[$. Its infimum is $a$, which is the minimum.

(11) Clearly, (a) $\Leftrightarrow$ (b) $\Rightarrow$ (c) $\Leftrightarrow$ (d). To prove (c) $\Rightarrow$ (b), suppose that $F(x, \varnothing, \infty)=1$ for all $x \in X$. Then, for all $A \in \mathcal{P}(X)$, by item (3), we have $F(x, A, \infty) \geq F(x, \varnothing, \infty)=1$.

The following properties refine the previous items in FA-spaces using property FA6.

Lemma 6.2 Let $(X, F)$ be a FA-space, $x \in X, t \geq 0$, and $A \in \mathcal{P}(X)$.

(1) $F(x, A, t)=F(x, \bar{A}, t)$ for all $t \in[0, \infty]$.

(2) $F(x, A, \cdot)$ is a nondecreasing function in $[0, \infty]$.

(3) $A$ is closed if, and only if, for each $x \in X$ such that $F(x, A, t)=1$ for all $t>0$, we have $x \in A$.

(4) $(X, F)$ satisfies property $F A 7$ if, and only if, points are closed subsets, that is, $\overline{\{x\}}=\{x\}$.

(5) $\left(A^{(r)}\right)^{(s)} \subseteq A^{(r+s)}$ for all $r, s \in[0, \infty]$.

(6) $A^{(r)}$ is closed for all $r \geq 0$.

(7) The closure $\bar{A}$ of $A$ is a closed set, that is, $\overline{\bar{A}}=\bar{A}$.

(8) $F(x, A, \infty)=F\left(x, A^{(r)}, \infty\right)$ for all $r \in[0, \infty]$.

Proof (1) As $A \subseteq \bar{A}$, item (3) of Lemma 6.1 implies that $F(x, A, t) \leq F(x, \bar{A}, t)$ for all $t \in[0, \infty]$. Let $t \in] 0, \infty[$ and let $\varepsilon \in] 0, t[$. Using FA6 with $s=\varepsilon>0=r$ and item (6) of Lemma 6.1

$$
F(x, A, t)=F(x, A, t-\varepsilon+\varepsilon) \geq F\left(x, A^{(0)}, t-\varepsilon\right) \geq F(x, A, t-\varepsilon) .
$$

As $F(x, A, \cdot)$ is left-continuous in $t, \lim _{\varepsilon \rightarrow 0^{+}} F(x, A, t-\varepsilon)=F(x, A, t)$, and as $F(x, \bar{A}, \cdot)$ is leftcontinuous in $t$ too, we deduce that

$$
\begin{gathered}
F(x, A, t) \geq \lim _{\varepsilon \rightarrow 0^{+}} F(x, \bar{A}, t-\varepsilon) \geq \lim _{\varepsilon \rightarrow 0^{+}} F(x, A, t-\varepsilon) \\
\Rightarrow \quad F(x, A, t) \geq F(x, \bar{A}, t) \geq F(x, A, t) .
\end{gathered}
$$

Finally, by FA6 with $r=0$ and $t=\infty$, we have $F(x, A, \infty) \geq F\left(x, A^{(0)}, \infty\right)=F(x, \bar{A}, \infty)$.

(2) Let $t \in[0, \infty[$ and $s \in] 0, \infty[$ be arbitrary numbers. Items (3) and (6) of Lemma 6.1 imply that, as $A \subseteq A^{(s / 2)}, F(x, A, t) \leq F\left(x, A^{(s / 2)}, t\right)$. Property FA6 implies that $F(x, A, t+s) \geq$ $F\left(x, A^{(s / 2)}, t\right) \geq F(x, A, t)$. Finally, by FA6 with $r=0$, we have $F(x, A, \infty) \geq F\left(x, A^{(0)}, t\right)=$ $F(x, \bar{A}, t)=F(x, A, t)$. Therefore, $F(x, A, \cdot)$ is a nondecreasing function in $[0, \infty]$.

(4) Suppose that $(X, F)$ satisfies the condition FA7. Let $y \in \overline{\{x\}}$, that is, $F(y, x, t)=1$ for all $t>0$. So $y=x$. The converse is similarly true.

(5) Suppose that $r, s \in\left[0, \infty\left[\right.\right.$ and $x \in\left(A^{(r)}\right)^{(s)}$, that is, $F\left(x, A^{(r)}, t\right)=1$, for all $\left.\left.t \in\right] s, \infty\right]$. If $z \in] r+s, \infty[$, there exists $\varepsilon>0$ such that $z>r+s+2 \varepsilon$, and then

$$
F(x, A, z) \geq F(x, A, r+s+2 \varepsilon) \geq F\left(x, A^{(r)}, s+\varepsilon\right)=1,
$$

so $F(x, A, z)=1$ for all $z \in] r+s, \infty]$. This means that $x \in A^{(r+s)}$. Other cases can be proved in a similar way. See Remark A.5 in the Appendix. 
(6) $\overline{A^{(r)}}=\left(A^{(r)}\right)^{(0)} \subseteq A^{(r+0)}=A^{(r)}$.

(7) $\overline{\bar{A}}=\left(A^{(0)}\right)^{(0)} \subseteq A^{(0+0)}=A^{(0)}=\bar{A}$.

(8) Taking into account that $A \subseteq A^{(r)}, F(x, A, t) \leq F\left(x, A^{(r)}, t\right)$ for all $t \in[0, \infty]$. The opposite inequality follows from the condition FA6.

Property (8) of the previous lemma is not intuitive. Property (6) of Lemma 6.1 means that the family of closures $\left\{A^{(r)}\right\}_{r \geq 0}$ is increasing. However, $F$ associates the same value to all closures when $t=\infty$. Although it can be less than 1 , the following proposition proves that it is natural that this number takes the value 1 .

Proposition 6.1 Let $(X, F)$ be a FA-space and define the fuzzy set $F_{1}: X \times \mathcal{P}(X) \times[0, \infty] \rightarrow$ $[0,1]$, for all $x \in X$, all $A \in \mathcal{P}(X)$ and all $t \in[0, \infty]$, by:

$$
F_{1}(x, A, t)= \begin{cases}F(x, A, t), & \text { if } t<\infty, \\ 1, & \text { if } t=\infty .\end{cases}
$$

Then $\left(X, F_{1}\right)$ is a FA-space, $F \leq F_{1}$ and $A_{1}^{(\infty)}=X$ for all $A \in \mathcal{P}(X)$.

Proof FA1 to FA5 are trivial. To prove FA6, if $t=\infty$ or $s=\infty$, then $F_{1}(x, A, t+s)=1 \geq$ $F_{1}\left(x, A_{1}^{(r)}, t\right)$. If $t, s \in[0, \infty[$ and $r \in[0, s[$, as $F(y, A, \cdot)$ is a nondecreasing function in $[0, \infty]$, then

$$
\begin{aligned}
A_{1}^{(r)} & \left.\left.=\left\{y \in X / F_{1}(y, A, t)=1, \forall t \in\right] r, \infty\right]\right\}=\{y \in X / F(y, A, t)=1, \forall t \in] r, \infty[\} \\
& =\{y \in X / F(y, A, t)=1, \forall t \in] r, \infty]\}=A^{(r)} .
\end{aligned}
$$

$F_{1}$ verifies $F_{1}(x, A, t+s)=F(x, A, t+s) \geq F\left(x, A^{(r)}, t\right)=F\left(x, A_{1}^{(r)}, t\right)=F_{1}\left(x, A_{1}^{(r)}, t\right)$.

\section{The induced topology in FA-spaces}

Let $(X, F)$ be a $\mathrm{F}_{s} \mathrm{~A}$-space. A subset $A \in \mathcal{P}(X)$ is open (w.r.t. $\left.F\right)$ if its complementary $X \backslash A$ is closed (w.r.t. $F$ ). The subsets $\varnothing$ and $X$ are clearly open (w.r.t. $F$ ). Now consider the family of all open subsets of $X$ :

$$
\Upsilon_{F}=\{X \backslash \bar{A} / A \in \mathcal{P}(X)\}
$$

To prove that $\Upsilon_{F}$ is, in some cases, a topology on $X$, the following result is needed.

Proposition 7.1 Let $(X, F)$ be a $F_{s} A$-space, $s \in[0, \infty]$ and $A, B \in \mathcal{P}(X)$ subsets of $X$.

(1) $A^{(s)} \cup B^{(s)} \subseteq(A \cup B)^{(s)}$.

(2) If $(X, F)$ verifies the equality in $F A 5$ and $F(x, A, \cdot)$ and $F(x, B, \cdot)$ are nondecreasing in $[0, \infty]$, then $(A \cup B)^{(s)}=A^{(s)} \cup B^{(s)}$.

Proof (1) As $A \subseteq A \cup B$ and $B \subseteq A \cup B$, item (5) of Lemma 6.1 implies $A^{(s)} \subseteq(A \cup B)^{(s)}$ and $B^{(s)} \subseteq(A \cup B)^{(s)}$, and then $A^{(s)} \cup B^{(s)} \subseteq(A \cup B)^{(s)}$.

(2) Suppose that $(X, F)$ verifies the equality in FA5 and $F(x, A, \cdot)$ and $F(x, B, \cdot)$ are nondecreasing. Let $x \in(A \cup B)^{(s)}$ be any point. If $s=\infty$, then $\max (F(x, A, \infty), F(x, B, \infty))=$ 
$F(x, A \cup B, \infty)=1$, and we see that $x \in A^{(\infty)}$, if $F(x, A, \infty)=1$, or $x \in B^{(\infty)}$, if $F(x, B, \infty)=1$. Suppose that $s<\infty$. In this case:

$$
\max (F(x, A, t), F(x, B, t))=F(x, A \cup B, t)=1 \quad \text { for all } t \in] s, \infty]
$$

As $\max (F(x, A, t), F(x, B, t))=1$ for all $t \in] s, \infty]$, we can define

$$
t_{1}=\inf (\{s \in[0, \infty[/ F(x, A, s)=1\})
$$

and $t_{2}=\inf \left(\left\{s \in[0, \infty[/ F(x, B, s)=1\})\right.\right.$, and we have $t_{1}<\infty$ or $t_{2}<\infty$ (even if one of the two sets is empty; the other set is a non-empty and non-bounded real interval since $F(x, A, \cdot)$ and $F(x, B, \cdot)$ are nondecreasing). Let $t_{0}=\min \left(t_{1}, t_{2}\right)<\infty$. If $t_{0}>s$, there exists $\left.r \in\right] s, t_{0}[$. As $r>s$, then $\max (F(x, A, r), F(x, B, r))=1$, so $F(x, A, r)=1$ or $F(x, B, r)=1$. If $F(x, A, r)=1$, then $t_{1} \leq r$ and if $F(x, B, r)=1, t_{2} \leq r$. In any case, $t_{0}=\min \left(t_{1}, t_{2}\right) \leq r$, but this contradicts $t_{0}>r$. So $t_{0} \leq s$. If $t_{0}=t_{1} \leq s$, then $F(x, A, t)=1$ for all $t>t_{1} \geq s$, so $x \in A^{(s)}$. If $t_{0}=t_{2} \leq s$, then $F(x, B, t)=1$ for all $t>t_{2} \geq s$, so $x \in B^{(s)}$. In any case, $x \in A^{(s)} \cup B^{(s)}$.

Theorem 7.1 Every FA-space $(X, F)$ satisfies the following properties:

(1) $\varnothing, X \in \Upsilon_{F}$.

(2) The arbitrary intersection of closed subsets of $X$ is also a closed subset of $X$.

(3) If $(X, F)$ reaches the equality in FA5, then a finite union of closed subsets of $X$ is also a closed subset of $X$.

Proof (2) Let $\left\{A_{i}\right\}_{i \in I}$ be a family of closed subsets of $X$ and let $B=\cap_{i \in I} A_{i}$ be the intersection. If $B=\varnothing, B$ is closed. Suppose that $B \neq \varnothing$ and let $x \in \bar{B}$ be a point. This is equivalent to $F(x, B, t)=1$ for all $t>0$. As $B \subseteq A_{j}$, for each $j \in I$, item (3) of Lemma 6.1 implies $F\left(x, A_{j}, t\right) \geq F(x, B, t)=1$ for all $t>0$. As $F\left(x, A_{j}, t\right)=1$ for all $t>0$, we have $x \in \overline{A_{j}}=A_{j}$ for all $j \in I$. So $x \in \cap_{i \in I} A_{i}=B$ and $B$ is closed.

(3) Let $A_{1}, A_{2} \subseteq X$ be two closed and non-empty subsets of $X$. From item (2) of Proposition 7.1, we have $\overline{A_{1} \cup A_{2}}=\left(A_{1} \cup A_{2}\right)^{(0)}=A_{1}^{(0)} \cup A_{2}^{(0)}=\overline{A_{1}} \cup \overline{A_{2}}=A_{1} \cup A_{2}$, so $A_{1} \cup A_{2}$ is closed. The rest can be proved by induction.

Taking the complement in $X$, the previous result has the following corollary.

Corollary 7.1 If an FA-space $(X, F)$ verifies the equality in FA5 axiom, then the family $\Upsilon_{F}$ is a topology on $X$.

In fact, for all $x \in X$, the collection $\beta_{x}=\left\{\{x\}^{(r)}, r>0\right\}$ is a basis of neighborhoods of $\Upsilon_{F}$ at the point $x$.

See Proposition A.6 in the Appendix.

If $(X, F)$ is an FA-space which verifies the equality in the FA5 axiom, the (fuzzy) topology in $X$ induced by the fuzzy metric $F$ is the topology $\Upsilon_{F}$ defined in Corollary 7.1.

\section{Fixed point theory in FA-spaces}

In this section, in order to show that the category of FA-spaces is appropriate to study fixed point theorems, we present a simple but interesting result in this setting. Its main advantage is that it can be particularized at the same time to the categories of metric 
spaces, quasi-metric spaces, approach spaces, and fuzzy metric spaces (both in the sense of Kramosil and Michálek and in the sense of George and Veeramani). Before that, we present the following notions, which are the natural definitions associated to the topology $\Upsilon_{F}$. Notice that $F$ is not necessarily symmetric, so we must distinguish right and left notions.

Definition 8.1 Let $(X, F)$ be a $\mathrm{F}_{s} \mathrm{~A}$-space, let $x \in X$ be a point and let $\left\{x_{n}\right\} \subseteq X$ be a sequence. We say that $\left\{x_{n}\right\}$ is a:

- right-Cauchy sequence if for all $r>0$, there exists $n_{0} \in \mathbb{N}$ such that if $m>n \geq n_{0}$, then $x_{n} \in\left\{x_{m}\right\}^{(r)}$, in the sense that

$$
F\left(x_{n}, x_{m}, t\right)=1 \quad \text { for all } t>r
$$

- left-Cauchy sequence if for all $r>0$, there exists $n_{0} \in \mathbb{N}$ such that if $m>n \geq n_{0}$, then $x_{m} \in\left\{x_{n}\right\}^{(r)}$, in the sense that

$$
F\left(x_{m}, x_{n}, t\right)=1 \quad \text { for all } t>r
$$

- Cauchy sequence if it is both right-Cauchy and left-Cauchy;

- convergent sequence to $x$ if, for all $r>0$, there exists $n_{0} \in \mathbb{N}$ such that if $n \geq n_{0}$, then $x_{n} \in\{x\}^{(r)}$ and $x \in\left\{x_{n}\right\}^{(r)}$.

We say that $(X, F)$ is complete if every Cauchy sequence in $(X, F)$ is convergent to a point of $X$.

Remark 8.1 Bound and semi-bound sets in FM-space are usually introduced as follows. Given a non-empty subset $A$ of a $\mathrm{F}_{q} \mathrm{M}$-space $(X, M, *)$, let $\varphi_{A}$ the function from $[0, \infty[$ into $[0,1]$ defined by

$$
\varphi_{A}(t)=\inf \{M(x, y, t): x, y \in A\} .
$$

As $\varphi_{A}$ is nondecreasing on [0, $\infty$, it generates a unique left-continuous function $D_{A}$ from $[0, \infty[$ into $[0,1]$ given by

$$
D_{A}(0)=0 \quad \text { and } \quad D_{A}(t)=\lim _{s \rightarrow t^{-}} \varphi_{A}(s) \quad \text { for all } t>0
$$

The function $D_{A}$ is known as the probabilistic diameter of $A$. Following [3], the subset $A$ is bounded if $\lim _{t \rightarrow \infty} D_{A}(t)=1$, and it is semibounded if $0<\lim _{t \rightarrow \infty} D_{A}(t)<1$.

Notice that a subset only containing a pair of points of $X$ must be bounded. But two possibilities arise when a M-space is seen as a FM-space. From a probabilistic point of view, (2) shows that $D_{A}(t)$ does not take the value 1 when $t \in[0, \infty[$. But, from a deterministic point of view, it seems to be reasonable that, when $A$ is bounded, there must exist a point $t_{0}$ such that $P\left(d(a, b) \leq t_{0}\right)=1$ for all $a, b \in A$. In fact, our way to embed qM-spaces as FMspaces is closer to the second view-point (see Lemma 2.2). Hence, in the following result, the existence of $x_{0}$ seems to be natural, because the set $\left\{x_{0}, T x_{0}\right\}$ must be bounded.

Next, we present the main result of this section. 
Theorem 8.1 Let $(X, F)$ be a complete FA-space verifying condition $F A 7$ and let $T: X \rightarrow X$ be a self-mapping such that there exists $\lambda \in(0,1)$ satisfying

$F(T x, T A, \lambda t) \geq F(x, A, t) \quad$ for all $x \in X$, all $A \in \mathcal{P}(X) \backslash\{\varnothing\}$ and all $t>0$.

If there exist $x_{0} \in X$ and $t_{0} \in(0, \infty)$ such that $F\left(x_{0}, T x_{0}, t_{0}\right)=F\left(T x_{0}, x_{0}, t_{0}\right)=1$, then $T$ has, at least, a fixed point.

Furthermore, if for all $x, y \in \operatorname{Fix}(T)$ we have $\lim _{t \rightarrow \infty} F(x, y, t)=1$, then $T$ has a unique fixed point.

Notice that, throughout the proof, we will only need that

$$
F(T x, T y, \lambda t) \geq F(x, y, t)
$$

for all $x, y \in X$ and all $t>0$. We also point out that the hypothesis for uniqueness is coherent with Kramosil and Michálek's original notion (recall (1)).

Proof Given the points $x_{0} \in X$ and $t_{0} \in(0, \infty)$ such that $F\left(x_{0}, T x_{0}, t_{0}\right)=F\left(T x_{0}, x_{0}, t_{0}\right)=1$, let us define

$$
x_{n+1}=T x_{n} \quad \text { and } \quad t_{n+1}=\lambda t_{n} \quad \text { for all } n \in \mathbb{N} .
$$

Using the fact that $F\left(x_{0}, T x_{0}, t_{0}\right)=1$, we are going to show that $\left\{x_{n}\right\}$ is a right-Cauchy sequence in $(X, F)$. In a similar way, the reader can prove that $\left\{x_{n}\right\}$ is a left-Cauchy sequence in $(X, F)$ employing that $F\left(T x_{0}, x_{0}, t_{0}\right)=1$.

Notice that as $t_{0} \in(0, \infty)$ and $\lambda \in(0,1)$, then

$$
\left\{t_{n}\right\} \rightarrow 0^{+} \quad \text { and } \quad t_{n+1}<t_{n} \quad \text { for all } n \in \mathbb{N} \text {. }
$$

Moreover, the series

$$
\sum_{n \in \mathbb{N}} t_{n}=\sum_{n \in \mathbb{N}} \lambda^{n-1} t_{0}=t_{0} \sum_{n \in \mathbb{N}} \lambda^{n-1}
$$

is geometric, so it is convergent. We claim that

$$
F\left(x_{n}, x_{n+1}, t_{n}\right)=1 \quad \text { for all } n \in \mathbb{N} \text {. }
$$

Indeed, for $n=0$, it is obvious. Assume that (7) holds for some $n \geq 0$. Therefore,

$$
F\left(x_{n+1}, x_{n+2}, t_{n+1}\right)=F\left(T x_{n}, T x_{n+1}, \lambda t_{n}\right) \geq F\left(x_{n}, x_{n+1}, t_{n}\right)=1 .
$$

Hence (7) holds for all $n \in \mathbb{N}$. In particular, (7) means that

$$
x_{n} \in\left\{x_{n+1}\right\}^{\left(t_{n}\right)} \quad \text { for all } n \in \mathbb{N} \text {. }
$$


We claim that

$$
x_{n} \in\left\{x_{n+m}\right\}^{\left(t_{n}+t_{n+1}+\cdots+t_{n+m-1}\right)} \quad \text { for all } n, m \in \mathbb{N} \text { with } m \geq 1 .
$$

We proceed by induction on $m$. If $m=1$, then (10) reduces to (7). Assume that (10) holds for some $m$ and we will prove it for $m+1$. Indeed, by (9), we have

$$
x_{n+m} \in\left\{x_{n+m+1}\right\}^{\left(t_{n+m}\right)} .
$$

If we join this fact with the hypothesis of induction (10), and using item (5) of Lemma 6.2, we deduce that

$$
\begin{aligned}
& \left.\begin{array}{l}
x_{n+m} \in\left\{x_{n+m+1}\right\}^{\left(t_{n+m}\right)} \\
x_{n} \in\left\{x_{n+m}\right\}^{\left(t_{n}+t_{n+1}+\cdots+t_{n+m-1}\right)}
\end{array}\right\} \\
& \Rightarrow \quad x_{n} \in\left\{x_{n+m}\right\}^{\left(t_{n}+t_{n+1}+\cdots+t_{n+m-1}\right)} \subseteq\left(\left\{x_{n+m+1}\right\}^{\left(t_{n+m}\right)}\right)^{\left(t_{n}+t_{n+1}+\cdots+t_{n+m-1}\right)} \\
& \subseteq\left\{x_{n+m+1}\right\}^{\left(t_{n}+t_{n+1}+\cdots+t_{n+m-1}+t_{n+m}\right)} \text {. }
\end{aligned}
$$

Hence, the induction is completed and (10) holds. In particular,

$$
F\left(x_{n}, x_{n+m}, t\right)=1 \quad \text { for all } n, m \in \mathbb{N} \text { and for all } t>t_{n}+t_{n+1}+\cdots+t_{n+m-1} .
$$

Next, we show that $\left\{x_{n}\right\}$ is a right-Cauchy sequence. Let $r>0$ be arbitrary. By (6), as the series $\sum_{n \in \mathbb{N}} t_{n}$ is convergent, there exists $n_{0} \in \mathbb{N}$ such that

$$
\sum_{n=n_{0}}^{\infty} t_{n}<r
$$

Hence, for all $n, m \in \mathbb{N}$ such that $n \geq n_{0}$ and $m \geq 1$, it follows from item (6) of Lemma 6.1 that

$$
x_{n} \in\left\{x_{n+m}\right\}^{\left(t_{n}+t_{n+1}+\cdots+t_{n+m-1}\right)} \subseteq\left\{x_{n+m}\right\}^{(r)},
$$

which means that $\left\{x_{n}\right\}$ is a right-Cauchy sequence.

In a similar way, using the fact that $F\left(T x_{0}, x_{0}, t_{0}\right)=1$, it is possible to prove that $\left\{x_{n}\right\}$ is a left-Cauchy sequence in $(X, F)$. As a result, $\left\{x_{n}\right\}$ is a Cauchy sequence in $(X, F)$. As $(X, F)$ is complete, there exists $z \in X$ such that $\left\{x_{n}\right\} F$-converges to $z$, that is,

$$
\forall r>0, \exists n_{0} \in \mathbb{N}:\left(n \geq n_{0} \Rightarrow x_{n} \in\{z\}^{(r)} \text { and } z \in\left\{x_{n}\right\}^{(r)}\right) .
$$

We claim that $z$ is a fixed point of $T$. To prove it, let $t>0$ be arbitrary. Since $\left\{x_{n}\right\} F$ converges to $z$, there exists $n_{0} \in \mathbb{N}$ such that

$$
x_{n} \in\{z\}^{(t / 4)} \text { and } z \in\left\{x_{n}\right\}^{(t / 4)} \text { for all } n \geq n_{0} .
$$

In particular, as

$$
\frac{t}{4}<\frac{t}{2}<\frac{t}{2 \lambda}
$$


and $z \in\left\{x_{n_{0}}\right\}^{(t / 4)}$, it follows that

$$
\begin{aligned}
F(T z, z, t) & \geq F\left(T z,\{z\}^{(t / 4)}, t / 2\right) \geq F\left(T z, x_{n_{0}+1}, t / 2\right) \\
& =F\left(T z, T x_{n_{0}}, t / 2\right) \geq F\left(z, x_{n_{0}}, t /(2 \lambda)\right)=1 .
\end{aligned}
$$

This demonstrates that $F(T z, z, t)=1$ for all $t>0$. By condition FA7, we conclude that $T z=z$, that is, $z$ is a fixed point of $T$.

Next assume that condition (U) holds and let $x, y \in \operatorname{Fix}(T)$ be two arbitrary fixed points of $T$. Therefore, for all $t>0$,

$$
F(x, y, t)=F(T x, T y, t) \geq F(x, y, t / \lambda)
$$

By induction, we obtain, for all $t>0$,

$$
F(x, y, t) \geq F\left(x, y, \frac{t}{\lambda^{n}}\right) \quad \text { for all } n \in \mathbb{N} .
$$

Letting $n \rightarrow \infty$, the sequence $\left\{t / \lambda^{n}\right\}$ positively diverges, so that

$$
F(x, y, t) \geq \lim _{n \rightarrow \infty} F\left(x, y, \frac{t}{\lambda^{n}}\right)=\lim _{s \rightarrow \infty} F(x, y, s)=1
$$

for all $t>0$. As a consequence, using condition FA7, we conclude that $x=y$, which means that $T$ has a unique fixed point.

The first two arguments of $F$ are intrinsically different. Hence, it makes no sense to impose the requirement that $F$ is symmetric. However, to compensate the lack of symmetry in $F$, we introduce the following condition.

Definition 8.2 Given $M>0$, we say that a $\mathrm{F}_{s} \mathrm{~A}$-space $(X, F)$ is $M$-symmetric if $F(x, y, t) \leq$ $M F(y, x, t)$ for all $x, y \in X$ and all $t>0$.

Proposition 8.1 If $\left\{x_{n}\right\}$ is a sequence in a $M$-symmetric $F_{s} A$-space, then the following statements are equivalent.

(1) $\left\{x_{n}\right\}$ is a right-Cauchy sequence.

(2) $\left\{x_{n}\right\}$ is a left-Cauchy sequence.

(3) $\left\{x_{n}\right\}$ is a Cauchy sequence.

Theorem 8.2 Theorem 8.1 also holds if we replace the condition that there exist $x_{0} \in X$ and $t_{0} \in(0, \infty)$ such that $F\left(x_{0}, T x_{0}, t_{0}\right)=F\left(T x_{0}, x_{0}, t_{0}\right)=1$ by the following one:

$(\circledast)(X, F)$ is $M$-symmetric and there exist $x_{0} \in X$ and $t_{0} \in(0, \infty)$ such that $F\left(x_{0}, T x_{0}, t_{0}\right)=1$.

Proof Repeating, step by step, the arguments of the proof of Theorem 8.1, and using $F\left(x_{0}, T x_{0}, t_{0}\right)=1$, we deduce that $\left\{x_{n}\right\}$ is a right-Cauchy sequence in $(X, F)$. As $(X, F)$ is $M$-symmetric, Proposition 8.1 guarantees that $\left\{x_{n}\right\}$ is a Cauchy sequence in $(X, F)$. Then the proof of Theorem 8.1 can be repeated point by point. 
Example 8.1 Let $X$ be the set of all real numbers, $\mathbb{R}$, endowed with the Euclidean metric $\mathrm{d}_{E}(x, y)=|x-y|$ for all $x, y \in X$. Let us consider the mapping $F_{\mathrm{E}}: X \times \mathcal{P}(X) \times[0, \infty] \rightarrow[0,1]$ given, for all $x \in X$, all $A \in \mathcal{P}(X)$, and all $t \in[0, \infty]$, by

$$
F_{\mathrm{E}}(x, A, t)= \begin{cases}0, & \text { if } 0 \leq t \leq \delta_{\mathrm{d}}(x, A), \\ 1, & \text { if } \delta_{\mathrm{d}_{E}}(x, A)<t \text { or } t=\infty,\end{cases}
$$

where $\delta_{\mathrm{d}_{E}}$ is the approach metric defined in Lemma 2.1 corresponding to $\mathrm{d}_{E}$. Then $\left(X, F_{\mathrm{E}}\right)$ is a FA-space. Furthermore, as $\left(X, \mathrm{~d}_{E}\right)$ is complete, then $\left(X, F_{\mathrm{E}}\right)$ is also complete. Given $\lambda \in(0,1)$ and $\mu \in \mathbb{R}$, let $T: X \rightarrow X$ be the mapping $T x=\lambda x+\mu$ for all $x \in X$. Therefore, for all $x \in X$, all $A \in \mathcal{P}(X) \backslash\{\varnothing\}$, and all $t>0$,

$$
\begin{aligned}
F_{\mathrm{E}}(T x, T y, \lambda t) & =\left\{\begin{array}{ll}
0, & \text { if } 0 \leq \lambda t \leq \delta_{\mathrm{d}_{E}}(T x, T y), \\
1, & \text { if } \delta_{\mathrm{d}_{E}}(T x, T y)<\lambda t
\end{array}\right\}=\left\{\begin{array}{ll}
0, & \text { if } 0 \leq \lambda t \leq \mathrm{d}_{E}(T x, T y), \\
1, & \text { if } \mathrm{d}_{E}(T x, T y)<\lambda t
\end{array}\right\} \\
& =\left\{\begin{array}{ll}
0, & \text { if } 0 \leq \lambda t \leq|(\lambda x+\mu)-(\lambda y+\mu)|, \\
1, & \text { if }|(\lambda x+\mu)-(\lambda y+\mu)|<\lambda t
\end{array}\right\} \\
& =\left\{\begin{array}{ll}
0, & \text { if } 0 \leq \lambda t \leq \lambda|x-y|, \\
1, & \text { if } \lambda|x-y|<\lambda t
\end{array}\right\}=\left\{\begin{array}{ll}
0, & \text { if } 0 \leq t \leq|x-y|, \\
1, & \text { if }|x-y|<t
\end{array}\right\} \\
& =F_{\mathrm{E}}(x, y, t) .
\end{aligned}
$$

Then $T$ satisfies (5). Taking into account that every point $x_{0} \in X$ verifies $F\left(x_{0}, T x_{0}, t_{0}\right)=$ $F\left(T x_{0}, x_{0}, t_{0}\right)=1$ for all $t_{0}>\mathrm{d}_{E}\left(x_{0}, T x_{0}\right)$, then Theorem 8.1 guarantees that $T$ has a unique fixed point.

\section{Appendix: Some remarks about the proofs}

For the sake of completeness, this appendix includes the proofs of some statements used in this manuscript.

Proposition A.1 Let $d: X \times X \rightarrow[0, \infty]$ be a map. Let $f, g: X \times \mathcal{P}(X) \times[0, \infty] \rightarrow[0,1]$ be the crisp fuzzy sets of $X \times \mathcal{P}(X) \times[0, \infty]$ defined, for all $x \in X$, all $A \in \mathcal{P}(X)$, and all $t \in[0, \infty]$, as follows:

$$
\begin{aligned}
& \text { if } \inf _{a \in A} d(x, a)=\infty, \quad f(x, A, t)= \begin{cases}0, & \text { if } t<\infty, \\
1, & \text { if } t=\infty ;\end{cases} \\
& \text { if } \inf _{a \in A} d(x, a)<\infty, \quad f(x, A, t)= \begin{cases}0, & \text { if } t \leq \inf _{a \in A} d(x, a), \\
1, & \text { if } \inf _{a \in A} d(x, a)<t ;\end{cases} \\
& g(x, a, t)= \begin{cases}1, & \text { if } d(x, a)<t<\infty \text { or } t=\infty, \\
0, & \text { in any other case; }\end{cases} \\
& g(x, A, t)= \begin{cases}0, & \text { if } A=\varnothing \text { and } t<\infty, \\
1, & \text { if } A=\varnothing \text { and } t=\infty, \\
\sup _{a \in A} g(x, a, t), & \text { if } A \neq \varnothing,\end{cases}
\end{aligned}
$$

considering $\inf _{a \in \varnothing} d(x, a)=\infty$. Then $f=g$. 
Proof If $A=\varnothing$, then

$$
f(x, \varnothing, t)=\left\{\begin{array}{ll}
0, & \text { if } t<\infty, \\
1, & \text { if } t=\infty
\end{array}\right\}=g(x, \varnothing, t) .
$$

Suppose that $A \neq \varnothing$. If $\inf _{a \in A} d(x, a)=\infty$, then $d(x, a)=\infty$ for all $a \in A$. Then

$$
f(x, A, t)=\left\{\begin{array}{ll}
0, & \text { if } t<\infty, \\
1, & \text { if } t=\infty
\end{array}\right\}=g(x, A, t)
$$

Now suppose that $\inf _{a \in A} d(x, a)<\infty$. Then

$$
\begin{aligned}
& f(x, A, t)=\left\{\begin{array}{ll}
0, & \text { if } t \leq \inf _{a \in A} d(x, a), \\
1, & \text { if } \inf _{a \in A} d(x, a)<t \leq \infty
\end{array}\right. \text { and } \\
& g(x, A, t)=\sup _{a \in A} \begin{cases}0, & \text { if } t<d(x, a)=\infty \text { or } t \leq d(x, a)<\infty, \\
1, & \text { if } t=\infty \text { or } d(x, a)<t<\infty .\end{cases}
\end{aligned}
$$

Clearly, $f(x, A, 0)=0=g(x, A, 0)$ and $f(x, A, \infty)=1=g(x, A, \infty)$. Let $t \in] 0, \infty[$ be a real number.

If $0<t \leq \inf _{a \in A} d(x, a), f(x, A, t)=0$. For all $a \in A, t \leq \inf _{a \in A} d(x, a) \leq d(x, a)$, and the equality occurs if, and only, if, $d\left(x, a_{0}\right)=\inf _{a \in A} d(x, a)=t<\infty$. Then $g(x, A, t)=\sup _{a \in A} 0=$ $0=f(x, A, t)$.

If $t>\inf _{a \in A} d(x, a)$, then $f(x, A, t)=1$, and taking into account that $\inf _{a \in A} d(x, a)<t<\infty$, it follows that there exists $a_{0} \in A$ such that $d\left(x, a_{0}\right)<t$. So $g(x, A, t)=1=f(x, A, t)$. In any case, $f=g$.

Remark A.2 (FA5 in Proposition 4.1) If $\delta(x, A \cup B)=\infty$, then $\delta(x, A)=\delta(x, B)=\infty$ and

$$
\begin{aligned}
\max \left(F_{\delta}(x, A, t), F_{\delta}(x, B, t)\right) & =\max \left(\left\{\begin{array}{ll}
0, & \text { if } t<\infty, \\
1, & \text { if } t=\infty
\end{array}\right\},\left\{\begin{array}{ll}
0, & \text { if } t<\infty, \\
1, & \text { if } t=\infty
\end{array}\right\}\right) \\
& =\left\{\begin{array}{ll}
0, & \text { if } t<\infty, \\
1, & \text { if } t=\infty
\end{array}\right\}=F_{\delta}(x, A \cup B, t) .
\end{aligned}
$$

If $\delta(x, A \cup B)=\delta(x, A)<\infty=\delta(x, B)$, then

$$
\begin{aligned}
\max \left(F_{\delta}(x, A, t), F_{\delta}(x, B, t)\right) & =\max \left(\left\{\begin{array}{ll}
0, & \text { if } t \leq \delta(x, A)<\infty, \\
1, & \text { if } \delta(x, A)<t<\infty
\end{array}\right\},\left\{\begin{array}{ll}
0, & \text { if } t<\infty, \\
1, & \text { if } t=\infty
\end{array}\right\}\right) \\
& =\left\{\begin{array}{ll}
0, & \text { if } t \leq \delta(x, A)<\infty \\
1, & \text { if } \delta(x, A)<t<\infty
\end{array}\right\}=F_{\delta}(x, A \cup B, t) .
\end{aligned}
$$

Remark A.3 (FA6 in Proposition 4.1) If $t=\infty$ or $s=\infty$, then $F_{\delta}(x, A, t+s)=F_{\delta}(x, A, \infty)=$ 1 , and this value is trivially greater than or equal to $F_{\delta}\left(x, A^{(r)}, \infty\right)$.

Remark A.4 (item (6) of Lemma 6.1) For the cases $r<\infty$ and $s=\infty$, given a point $x \in$ $A^{(r)}$, it follows that $F(x, A, t)=1$ for all $\left.\left.t \in\right] r, \infty\right]$. As $F(x, A, \infty)=1$, then $x \in A^{(\infty)}=A^{(s)}$. If $r=s=\infty$, the result is obvious. 
Remark A.5 (item (5) of Lemma 6.2) If $r=\infty$ and $s<\infty$, clearly $A^{(\infty)} \subseteq\left(A^{(\infty)}\right)^{(s)}$. If $x \in$ $\left(A^{(\infty)}\right)^{(s)}, F\left(x, A^{(\infty)}, t\right)=1$ for all $t>s$. Using FA6, $F(x, A, \infty) \geq F\left(x, A^{(\infty)}, s+1\right)=1$, so $x \in$ $A^{(\infty)}$.

If $r<\infty$ and $s=\infty$, as $A \subseteq A^{(r)}$, then $A^{(\infty)} \subseteq\left(A^{(r)}\right)^{(\infty)}$ by item (6) of Lemma 6.1. Conversely, let $x \in\left(A^{(r)}\right)^{(\infty)}$, that is, $F\left(x, A^{(r)}, \infty\right)=1$. By FA6 with $t=s=\infty, F(x, A, \infty) \geq$ $F\left(x, A^{(r)}, \infty\right)=1$, so $x \in A^{(\infty)}$.

If $r=s=\infty$ and $x \in\left(A^{(\infty)}\right)^{(\infty)}$, then $F\left(x, A^{(\infty)}, \infty\right)=1$, and by FA6, $F(x, A, \infty) \geq$ $F\left(x, A^{(\infty)}, \infty\right)=1$, so $x \in A^{(\infty)}$.

Proposition A.6 (see [42]) Let $X$ be a set and, for all $x \in X$, let $\beta_{x}$ be a non-empty family of subsets of $X$ verifying the following properties:

(1) $x \in N$ for all $N \in \beta_{x}$.

(2) For all $N_{1}, N_{2} \in \beta_{x}$, there exists $N_{3} \in \beta_{x}$ such that $N_{3} \subseteq N_{1} \cap N_{2}$.

(3) For all $N \in \beta_{x}$, there exists $N^{\prime} \in \beta_{x}$ such that for all $y \in N^{\prime}$, there exists $N^{\prime \prime} \in \beta_{y}$ verifying $N^{\prime \prime} \subseteq N$.

Then there exists a unique topology $\tau$ on $X$ such that $\beta_{x}$ is a neighborhood system at $x$.

Competing interests

The authors declare that they have no competing interests.

Authors' contributions

All authors contributed equally and significantly in writing this article. All authors read and approved the final manuscript.

\section{Author details}

'University of Jaén, Campus las Lagunillas s/n, Jaén, 23071, Spain. ${ }^{2}$ Institute of Research and Development of Processes IIDP, Faculty of Science and Technology, University of the Basque Country, Campus of Leioa (Bizkaia), Barrio Sarriena, P.O. Box 644, Bilbao, 48940, Spain. ${ }^{3}$ Department of Statistics and Operations Research, University of Granada, Campus Fuentenueva, Granada, Spain.

\section{Acknowledgements}

The authors are very grateful to the Spanish Government for Grant DPI2012-30651 and to the Basque Government for Grant IT378-10. This paper has been partially supported by projects FQM-178, FQM-268 and FQM-265 of the Andalusian CICE, Spain.

Received: 15 November 2014 Accepted: 30 January 2015 Published online: 27 February 2015

\section{References}

1. Menger, K: Statistical metrics. Proc. Natl. Acad. Sci. USA 28, 535-537 (1942)

2. Zadeh, LA: Fuzzy sets. Inf. Control 8, 338-353 (1965)

3. Schweizer, B, Sklar, A: Probabilistic Metric Spaces. Dover, New York (2005)

4. Erceg, MA: Metric spaces in fuzzy set theory. J. Math. Anal. Appl. 69, 205-230 (1979)

5. Kaleva, O, Seikkala, S: On fuzzy metric spaces. Fuzzy Sets Syst. 12, 215-229 (1984)

6. Kramosil, I, Michálek, J: Fuzzy metrics and statistical metric spaces. Kybernetika 11, 336-344 (1975)

7. George, A, Veeramani, P: On some results in fuzzy metric spaces. Fuzzy Sets Syst. 64, 395-399 (1994)

8. Park, JH: Intuitionistic fuzzy metric spaces. Chaos Solitons Fractals 22, 1039-1046 (2004)

9. Grabiec, MT, Cho, YJ, Saadati, R: Families of quasi-pseudo-metrics generated by probabilistic quasi-pseudo-metric spaces. Surv. Math. Appl. 2, 123-143 (2007)

10. Rodríguez-López, J, Romaguera, S: The Hausdorff fuzzy metric on compact sets. Fuzzy Sets Syst. 147, $273-283$ (2004)

11. Deng, Z: Fuzzy pseudo metric spaces. J. Math. Anal. Appl. 86, 74-95 (1982)

12. Castro-Company, F, Romaguera, S: Experimental results for information system based on accesses locality via intuitionist fuzzy metrics. Open Cybern. Syst. J. 2, 158-172 (2008)

13. Colubi, A, González-Rodríguez, G: Triangular fuzzification of random variables and power of distribution tests: empirical discussion. Comput. Stat. Data Anal. 51, 4742-4750 (2007)

14. Aguilar-Peña, C, Roldán-López-de-Hierro, AF, Roldán-López-de-Hierro, C, Martínez-Moreno, J: A family of fuzzy distance measures of fuzzy numbers. Soft Comput. (2014). doi:10.1007/s00500-014-1497-0

15. Roldán, C, Roldán, A, Martínez-Moreno, J: A fuzzy regression model based on distances and random variables with crisp input and fuzzy output data: a case study in biomass production. Soft Comput. 16, 785-795 (2012)

16. Roldán, A, Martínez-Moreno, J, Roldán, C: Some applications of the study of the image of a fuzzy number: countable fuzzy numbers, operations, regression and a specificity-type ordering. Fuzzy Sets Syst. 257, 204-216 (2014)

17. Chauhan, S, Radenović, S, Imdad, M, Vetro, C: Some integral type fixed point theorems in non-Archimedean Menger PM-spaces with common property (E.A) and application of functional equations in dynamic programming. Rev. R. Acad. Cienc. Exactas Fís. Nat., Ser. A Mat. (2014). doi:10.1007/s13398-013-0142-6 
18. Chauhan, S, Bhatnagar, S, Radenović, S: Common fixed point theorems for weakly compatible mappings in fuzzy metric spaces. Matematiche 68(1), 87-98 (2013). doi:10.4418/2013.68.1.8

19. Van An, T, Van Dung, N, Kadelburg, Z, Radenović, S: Various generalizations of metric spaces and fixed point theorems. Rev. R. Acad. Cienc. Exactas Fís. Nat., Ser. A Mat. (2014). doi:10.1007/s13398-014-0173-7

20. Fang, JX: On fixed point theorem in fuzzy metric spaces. Fuzzy Sets Syst. 46, 107-113 (1992)

21. Fang, JX: A note on fixed point theorems of Hadžić. Fuzzy Sets Syst. 48, 391-395 (1992)

22. Golet, I: On contractions in probabilistic metric spaces. Rad. Mat. 13, 87-92 (2004)

23. Gregori, V, Sapena, A: On fixed point theorem in fuzzy metric spaces. Fuzzy Sets Syst. 125, 245-252 (2002)

24. Hadžić, O: Fixed point theorems for multi-valued mappings in some classes of fuzzy metric spaces. Fuzzy Sets Syst. 29, 115-125 (1989)

25. Hicks, TL: Fixed point theory in probabilistic metric spaces. Novi Sad J. Math. 13, 64 (1983)

26. Latif, A, Roldán-López-de-Hierro, AF, Sintunaravat, W: On common $\alpha$-fuzzy fixed points with applications. Fixed Point Theory Appl. 2014, 234 (2014)

27. Pap, E, Hadžić, O, Mesiar, R: A fixed point theorem in probabilistic metric spaces and an application. J. Math. Anal. Appl. 202, 433-449 (1996)

28. Razani, A, Shirdaryadzi, M: Some results on fixed points in fuzzy metric space. J. Appl. Math. Comput. 20, 401-408 (2006)

29. Roldán-López-de-Hierro, AF, Sintunaravat, W: Common fixed point theorems in fuzzy metric spaces using the CLRg property. Fuzzy Sets Syst. (2014). doi:10.1016/j.fss.2014.11.005

30. Roldán-López-de-Hierro, AF, Karapınar, E, Manro, S: Some new fixed point theorems in fuzzy metric spaces. J. Intell. Fuzzy Syst. 27, 2257-2264 (2014). doi:10.3233/IFS-141189

31. Roldán, A, Martínez-Moreno, J, Roldán, C, Cho, YJ: Multidimensional coincidence point results for compatible mappings in partially ordered fuzzy metric spaces. Fuzzy Sets Syst. 251, 71-82 (2014)

32. Alaca, C, Turkoglu, D, Yildiz, C: Fixed points in intuitionistic fuzzy metric spaces. Chaos Solitons Fractals 29(5), 1073-1078 (2006)

33. Chauhan, S, Shatanawi, W, Kumar, S, Radenović, S: Existence and uniqueness of fixed points in modified intuitionistic fuzzy metric spaces. J. Nonlinear Sci. Appl. 6, 1-12 (2013)

34. Martínez-Moreno, J, Roldán, A, Roldán, C: A note on the $\mathcal{L}$-fuzzy Banach's contraction principle. Chaos Solitons Fractals 41, 2399-2400 (2009)

35. Rafi, M, Noorani, MSM: Fixed point theorem on intuitionistic fuzzy metric spaces. Iran. J. Fuzzy Syst. 3, 23-29 (2006)

36. Saadati, R, Park, JH: On the intuitionistic fuzzy topological spaces. Chaos Solitons Fractals 27, 331-344 (2006)

37. Lowen, R: Approach spaces: a common supercategory of TOP and MET. Math. Nachr. 141, 183-226 (1989)

38. Lowen, R: Approach Spaces: The Missing Link in the Topology-Uniformity-Metric Triad. Oxford Mathematical Monographs. Oxford University Press, Oxford (1997)

39. Lowen, R, Sioen, M, Vaughan, D: Completing quasi-metric spaces - an alternative approach. J. Math. 29, 113-136 (2003)

40. Roldán, A, Martínez-Moreno, J, Roldán, C: On interrelationships between fuzzy metric structures. Iran. J. Fuzzy Syst. 10(2), 133-150 (2013)

41. Grabiec, M: Fixed points in fuzzy metric spaces. Fuzzy Sets Syst. 27, 385-389 (1988)

42. Willard, S: General Topology. Addison-Wesley Series in Mathematics. Dover, New York (2004)

\section{Submit your manuscript to a SpringerOpen ${ }^{\circ}$ journal and benefit from:}

- Convenient online submission

Rigorous peer review

- Immediate publication on acceptance

- Open access: articles freely available online

- High visibility within the field

- Retaining the copyright to your article 